\title{
Mobile Wellness Resources
}

Jennifer Herron, Indiana University School of Medicine, Indianapolis, IN

Stress in medical students is not unheard of nor is it not unexpected. Medical students have rigorous schedules and must pass a series of examinations that put an enormous amount of pressure on them to succeed. As medical students move into their career this same stress can follow them and impact not only their health, but that of their patients as well. This stress can lead to depression, anxiety and eventually cause burnout.

Libraries are already well aware of the stress students feel, especially around exam time. Therapy dogs visiting the libraries are a common sight and more libraries are beginning to offer some form of art therapy for students. Medical libraries can further support students' wellness needs is by promoting mobile wellness resources.

Keywords: Mobile applications, wellness, web resources, student stress

\section{INTRODUCTION}

It is not a secret that medical students and professionals face a large amount of stress in their lives. Medical students can become quickly overwhelmed and if they cannot find adequate coping methods their stress will only get worse which can lead to depression, anxiety, and burnout. Furthermore, if students do not learn how to manage their stress, it will continue to be a problem in their professional lives. Medical libraries have promoted a plethora of resources to

This is the author's manuscript of the article published in final edited form as:

Herron, J. (2016) Mobile Wellness Resources. Journal of Electronic Resources in Medical Libraries. 13(3) 93-98. http://dx.doi.org/10.1080/15424065.2016.1225542 
aid students in their medical education, and they should also consider the students' wellness and provide resources that can support them in managing their life, stress, and focusing on keeping themselves healthy. Mobile wellness resources are becoming more and more popular as technology continues to evolve and libraries can highlight these resources for students to aid in supporting student wellness.

\section{BACKGROUND}

Student stress is not a newfound discovery in medical schools and despite the schools best efforts, students are still experience high levels of stress. Medical schools began to address the issue of overstressed students in the 1970's and 1980's when stress management was introduced into the curriculum ${ }^{1}$. In the thirty-plus years that have since elapsed, more and more school sponsored resources have become available to students; however, these resources are not being as utilized as they should be. In an online survey study conducted on medical student awareness of wellness resources, $90 \%$ of students reported knowing about school resources available to them. This study also explored students' self-reported levels of stress and found that of those that struggled, only about $30 \%$ sought help to manage their stress ${ }^{2}$.

With resources readily available to them, why are medical students still not seeking support? A 2015 article published in the Student BMJ highlighted some of the reasons medical students fail to seek help when their stress levels may be rising. Stigma was one of the major reasons cited that students are reluctant to seek out help. Another reason described medical school culture as a contributing factor, which seems to be the exact opposite of what the schools are working to resolve. Twishaa Sheth, joint deputy chair of the Medical Students Committee of 
the British Medical Association, offered some insight on the problem, stating “...because of how competitive and difficult it is to get in, you're surrounded by very confident and outgoing people and can feel everyone around you is coping fine. That's a big reason people are not too keen on speaking out."3

While this problem cannot be solved overnight, wellness programs are being developed and started in medical schools across the country and throughout the world. More medical schools have begun to include mindfulness-based stress reduction as course electives, aiming to help students develop the skills needed to combat and manage stress. Additionally, grand rounds and other lecture series are being provided to students on topics that address methods of coping with stress and identifying the signs of burnout. ${ }^{4}$ Some schools have gone ever further and have trained faculty members who can provide guidance to students through Mind-Body Skills Groups.

After a review of 15 institutions with Mind-Body Skills Groups, it was found that after attending the skills groups students saw a reduction in stress levels, increased resilience and improved performance ${ }^{1}$. One key factor in the success of these Mind-Body Skills Groups is the ability to mesh with medical students busy schedules. As medical libraries most likely are aware, trying to plan an event and find a time that works with medical students schedules can be quite difficult.

\section{MOBILE WELLNESS RESOURCES}

One method to provide medical students with the support they need during a time that fits with their schedule would be through the use of mobile resources. A study of university students found that the most popular sources for health and wellness information were websites and 
mobile applications. ${ }^{5}$ Websites and mobile applications are part of a new wave of resources being used to reduce stress and promote wellness not only in students, but the general population as well. With these new types of interventions numerous studies have been conducted testing their effectiveness and impact on users. One such app, MoodHacker, was found to have significant impacts on users with depression, reducing their symptoms, decreasing negative thoughts, and increasing behavior changes. ${ }^{6}$ There are also websites available, such as MoodGym and BluePages, that offer a similar type of support to users and have shown similar promise at being a superior intervention tool. ${ }^{7}$

One award winning app that has been proven to be a highly successful intervention tool is the Virtual Hope Box. The Virtual Hope Box (VHB) is a combination of behavioral and cognitive therapy that has been successful in reducing stress in veterans. The VHB started off as an actual, physical box that was designed to combat veteran suicide and provide tailor-made support during times of stress and depression. While the physical hope boxes proved to be successful in helping veterans manage their stress, they did come with one disadvantage - they were not always easy to access. The physicality of the boxes meant that users would need to carry them with them if they wanted to have continuous access to them. Additionally, the box itself and its content did not provide the privacy that some users would have preferred. ${ }^{8}$ The Department of Defense National Center for Telehealth and Technology addressed these issues by creating the VHB app. In 2014 a study was conducted comparing the VHB to the traditional physical boxes. This study found the app to be hugely successful with over half of its study participants continuing to use the app after 6 weeks of usage. Furthermore, 100\% of participants reported that they would recommend the app to other service members and veterans. ${ }^{8}$ 
Another realm of mobile wellness resources that can be useful to medical students that address an underlying cause of stress are sleep apps. In addition to the academic stress that students face, is the effect that irregular hours can have on students' sleep health. The Scandinavian Journal of Work, Environment \& Health published a study which evaluated a mHealth intervention on airline pilots designed to improve their sleep quality, and subsequently, their overall health. The mHealth intervention focused around a mobile app called MORE energy. This was combined with a customized website that allowed pilots to enter their flight schedule and then choose to receive advice before, during, in-between, and after flights or receive specific advice on sleep and daylight exposure, nutrition, and physical activity. Almost $70 \%$ of pilots who participated in the study used the app and consequentially showed a reduction in fatigue and need for sleep-aids throughout their week. ${ }^{9}$ The MORE app was designed specifically for study participants and authors of the study concluded that providing a similar resource for people with irregular working hours would benefit them.

\section{EVALUATING MOBILE RESOURCES}

Not all mobile wellness resources have been evaluated in studies and published in research articles. While there are potentially a lot of beneficial resources available, there is also the potential for a lot of substandard apps. Trying to identify reputable mobile resources can be difficult, but librarians can utilize some of the same techniques that they teach patrons to use when evaluating information sources, with some leeway required. Reviewing author, owner, or developer information for these resources can yield some insight on the motivations for creating the resource and the background of knowledge that was applied when developing the content of these resources. Professional organizations will yield a more trustworthy resource, but private 
companies and individuals should not be excluded entirely without first searching for credible reviews. One strategy to use when trying to determine the quality of a resource is to search for it in databases such as PubMed. Reviews and research studies of popular mobile resources are becoming more common in PubMed and other medical databases. Additionally, performing general internet searches may also yield more reviews from medial or professional organizations.

Another method of evaluating mobile wellness resources would be to reach out to the offices and departments that provide student wellness support and work together to identify reliable resources. Staff members in these areas will most likely have the skill set to identify certain aspects or elements of the mobile resource that are useful for reducing and managing stress and improving overall wellness. Furthermore, by reaching out to these areas, libraries can form a unique partnership that directly benefits students.

\section{CONCLUSION}

Medical libraries provide their students with the tools and training to support their educational needs so they can become well-versed medical professionals. Librarians offer training on searching medical databases, provide guides on finding specific resources, and numerous other services to help students succeed. By highlighting mobile wellness resources, and furthermore offering space and computers for their use, libraries can provide students with support for managing their stress while also acting as an alternative outlet for health and wellness initiatives. Throughout their time in medical school, students learn to turn to the library for help finding resources. By expanding to include wellness in its breadth of subjects, libraries can become more active in supporting student wellness. 


\section{REFERENCES}

1. Gordon, J. S. (2014). Mind-body skills groups for medical students: reducing stress, enhancing commitment, and promoting patient-centered care. BMC Med Educ, 14, 198. doi:10.1186/1472-6920-14-198

2. Sayburn, A. (2015). Why medical students' mental health is a taboo subject: Why stigmas and misconceptions persist. Student BMJ. Retrieved from http://student.bmj.com/student/view-article.html?id=sbmj.h722

3. Walter, G., Soh, N. L., Norgren Jaconelli, S., Lampe, L., Malhi, G. S., \& Hunt, G. (2013). Medical students' subjective ratings of stress levels and awareness of student support services about mental health. Postgrad Med J, 89(1052), 311-315. doi:10.1136/postgradmedj-2012-131343

4. Slavin, S. J., Schindler, D. L., \& Chibnall, J. T. (2014). Medical student mental health 3.0: improving student wellness through curricular changes. Acad Med, 89(4), 573-577. doi:10.1097/acm.0000000000000166

5. Choi, W. and Stvilia, B. (2013), Use of mobile wellness applications and perception of quality. Proc. Am. Soc. Info. Sci. Tech., 50: 1-4. doi:10.1002/meet.14505001147

6. Birney, A. J., Gunn, R., Russell, J. K., \& Ary, D. V. (2016). MoodHacker Mobile Web App With Email for Adults to Self-Manage Mild-to-Moderate Depression: Randomized Controlled Trial. JMIR Mhealth Uhealth, 4(1), e8. doi:10.2196/mhealth.4231

7. Gilbody, S., Littlewood, E., Hewitt, C., Brierley, G., Tharmanathan, P., Araya, R., .. . White, D. (2015). Computerised cognitive behaviour therapy (cCBT) as treatment for depression in primary care (REEACT trial): large scale pragmatic randomised controlled trial. BMJ, 351, h5627. doi:10.1136/bmj.h5627

8. Bush, N. E., Dobscha, S. K., Crumpton, R., Denneson, L. M., Hoffman, J. E., Crain, A., . .. Kinn, J. T. (2015). A Virtual Hope Box smartphone app as an accessory to therapy: proof-of-concept in a clinical sample of veterans. Suicide Life Threat Behav, 45(1), 1-9. doi:10.1111/sltb.12103

9. van Drongelen, A., Boot, C. R., Hlobil, H., Twisk, J. W., Smid, T., \& van der Beek, A. J. (2014). Evaluation of an mHealth intervention aiming to improve health-related behavior and sleep and reduce fatigue among airline pilots. Scand J Work Environ Health, 40(6), 557-568. doi:10.5271/sjweh.3447 